Review

\title{
Exosomes in Mesenchymal Stem Cells, a New Therapeutic Strategy for Cardiovascular Diseases?
}

\author{
Lina Huang, Wenya Ma, Yidi Ma, Dan Feng, Hongyang Chen, Benzhi Cai ${ }^{\bowtie}$ \\ Department of Pharmacology, Harbin Medical University (the State-Province Key Laboratories of Biomedicine-Pharmaceutics of China), \\ Harbin 150081, China.
}

$\triangle$ Corresponding author: Dr. Benzhi Cai, Department of Pharmacology (the State-Province Key Laboratories of Biomedicine-Pharmaceutics of China), Harbin Medical University, 157\# Baojian Road, Nangang District, Harbin 150081, Heilongjiang, China; Tel.: +86 451 8666-9473; Fax: +86 451 8667-1354; Email: caibz@ems.hrbmu.edu.cn

C 2015 Ivyspring International Publisher. Reproduction is permitted for personal, noncommercial use, provided that the article is in whole, unmodified, and properly cited. Please see http:/ /ivyspring.com/terms for terms and conditions.

Received: 2014.10.04; Accepted: 2014.12.09; Published: 2015.01.12

\begin{abstract}
Cardiovascular diseases (CVDs) are still a major cause of people deaths worldwide, and mesenchymal stem cells (MSCs) transplantation holds great promise due to its capacity to differentiate into cardiovascular cells and secrete protective cytokines, which presents an important mechanism of MSCs therapy for CVDs. Although the capability of MSCs to differentiate into cardiomyocytes (CMCs), endothelial cells (ECs) and vascular smooth muscle cells (VSMCs) has been well recognized in massive previous experiments both in vitro and in vivo, low survival rate of transplanted MSCs in recipient hearts suggests that therapeutic effects of MSCs transplantation might be also correlated with other underlying mechanisms. Notably, recent studies uncovered that MSCs were able to secret cholesterol-rich, phospholipid exosomes which were enriched with microRNAs (miRNAs). The released exosomes from MSCs acted on hearts and vessels, and then exerted anti-apoptosis, cardiac regeneration, anti-cardiac remodeling, anti-inflammatory effects, neovascularization and anti-vascular remodeling, which are considered as novel molecular mechanisms of therapeutic potential of MSCs transplantation. Here we summarized recent advances about the role of exosomes in MSCs therapy for CVDs, and discussed exosomes as a novel approach in the treatment of CVDs in the future.
\end{abstract}

Key words: microRNA, exosomes, mesenchymal stem cell, myocardial infarction, reperfusion injury, pulmonary hypertension

\section{Introduction}

Cardiovascular diseases (CVDs) remain a major killer of human health and accord for the growing mortality and disability in developed countries and some developing countries such as China. Stem cells-based therapy showed great promise to regenerate damaged myocardium and treat CVDs. Embryonic stem cells (ESCs), induced pluripotent stem cells (iPSCs), endothelial progenitor cells (EPCs), mesenchymal stem cells (MSCs) and adipose tissue-derived stem cells (ADSCs) have shown therapeutic potential on CVDs and suggested as ideal sources of stem cells-based therapy [1]. In particular, MSCs transplantation has been proved as a new promising therapeutic approach for CVDs because MSCs possess multipotent differentiation ability and paracrine actions. MSCs originally derive from mesoderm and ectoderm during early embryonic development, and were found in various types of tissues and organs such as bone marrow [2], fat [3], muscles [4], lungs [5], liver [6], pancreas [7] and synovial membrane [8]. MSCs are capable to differentiate into osteoblasts [9], adipocytes [10], chondrocytes [11], CMCs [12], endothelial cells (ECs) [13] and vascular smooth muscle cells (VSMCs) [14] after the induction of certain microenvironments and cellular factors in vitro or in vivo. It has been widely recognized that 
engrafted MSCs differentiating into CMCs at least to a certain degree accounts for therapeutic effects of MSCs transplantation [15]. In addition to cardiomyocyte differentiation, MSCs are also capable to differentiate into ECs and VSMCs, which contributed to revascularization [16-18]. Accordingly, transplanted MSCs can replace the loss of vascular cells and CMCs in myocardial infarction (MI) and heart failure, and improved cardiac function of damaged hearts [19-21]. Whereas actually, it has been observed that only a minority of MSCs survived and engrafted after transplantation, and most of MSCs do not differentiate into CMCs, ECs and VSMCs to exert potent therapeutic effects. It suggests that MSCs might exert biological functions through multiple mechanisms.

Increasing evidence demonstrated that MSCs also can secrete functional paracrine factors such as vascular endothelial growth factor (VEGF), insulin-like growth factor-1 and basic fibroblast growth factor to produce various protective effects such as promoting vascular regeneration [22], supporting hematopoiesis [23], repairing injured kidney [24], facilitating myocardial repair [25] and ameliorating cardiac remodeling [26]. Interestingly, recent study revealed that transplanted MSCs may secret abundant particles documented as exosomes, which not only may reduce tissue injury but also enhance tissue repair [27]. Exosomes are cholesterol-rich, phospholipid vesicles enriched with microRNAs (miRNAs) which have been well recognized to regulate gene expression in a post-transcriptional manner and play a prominent role in various pathological and physiological processes [28-29] (Fig. 1). In the light of protective influences of exosomes on cardiovascular system, it is proposed that exosomes secreted by MSCs will be presented as an ideal therapeutic target for CVDs in the near future.

\section{Exosomes}

\subsection{Concept and characteristic of exosomes}

Exosomes are defined as nanosized membrane vesicles with a diameter of $30-100 \mathrm{~nm}$ that origins from multivesicular endosomal and are released by cells into extracellular environment. It is different from the other extracellular vesicles (EVs)-microvesicles which has a diameter of 100-1000 nm and origins from plasma membrane. It has been reported that there are multiple contents in exosomes including cytokines, proteins, lipids, mRNAs, miRNAs and ribosomal RNAs [30-31]. Exosomes can be released by various cells including $\mathrm{T}$ cells [32], B cells [33], reticulocytes [34], mast cells [35], platelets [36] and tumor cells [37-38]. For instance, miR-126 secreted from chronic myelogenous leuke- mia cells was found shuttled into endothelial cells, which modulated adhesive and migratory abilities of chronic myelogenous leukemia cells [39]. The exosomes derived from chronic myelogenous leukemia stimulated interleukin-8 (IL-8) release from bone marrow stromal cells, and promoted the progression of leukemia [40]. Thus, exosomes are suggested as central mediators of intercellular communication by transferring proteins, mRNAs and miRNAs to adjacent cells leading to coordinative function in organisms [41-42].

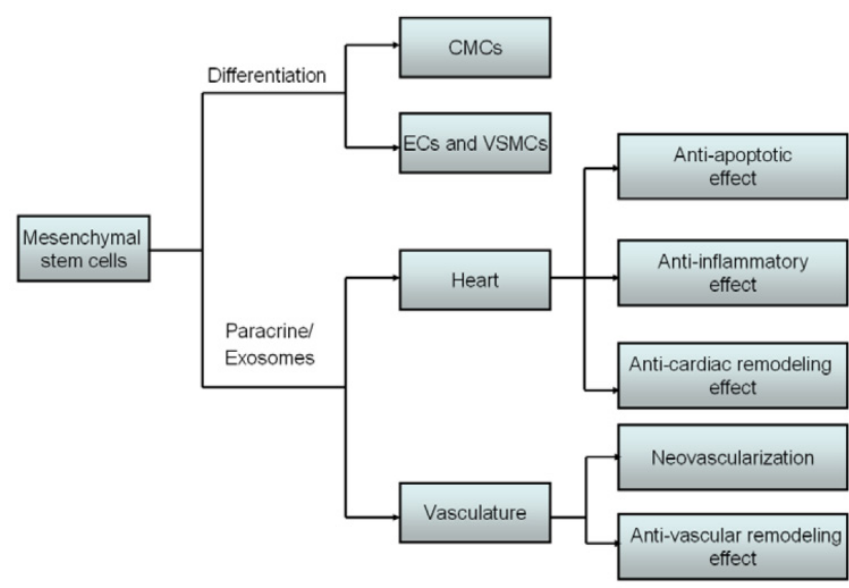

Fig. 1. Mechanisms of mesenchymal stem cells transplantation for CVDs. MSCs can differentiate into CMCs, ECs and VSMCs to replace the loss of cardiovascular cells. Besides, MSCs also secreted paracrine factors and exosomes to target heart and vasculature to exert anti-apoptosis, anti-cardiac remodeling, anti-inflammatory reactions, neovascularization and anti-vascular remodeling effects.

\subsection{Exosomes secreted by MSCs}

Likewise, MSCs also synthesize and secrete functional exosomes that are cholesterol-rich phospholipid vesicles. For example, bone marrow-derived MSCs may release the exosomes that can promote breast cancer cell dormancy in a metastatic niche [43]. Adipose MSCs secret the exosomes and microvesicles, and in turn regulate angiogenic potential of MSCs [44]. Gastric cancer tissue-derived MSCs also may release exosomes [45]. Moreover, injection of exosomes from MSCs into stroke rats might relieve symptoms by promoting angiogenesis, neurite remodeling and neurogenesis [46]. The exosomes derived from MSCs contain lipids, protein, mRNAs, precursor miRNAs (pre-miRNAs), miRNAs, etc. In particular, miRNAs in microparticles were sensitive to RNase only with the existence of phospholipase A2, sodium dodecyl sulfate-based cell lysis buffer or cyclodextrin, which definitely indicates that direct protection of RNAs against RNase was afforded by cholesterol-rich phospholipid vesicles [29]. 


\section{3. miRNAs mediated functions of exosomes}

It is well known that miRNAs play an essential role in various physiological and pathological processes by regulating gene expression at the post-transcription level [47]. The primary miRNAs (pri-miRNAs) are transcribed by RNA polymerase II and then are excised into pre-miRNAs by Drosha. Then, pre-miRNAs are cleaved by Dicer into mature miRNAs that assemble with argonaute (Ago) protein to form miRNA-induced silencing complex (RISC). It was recently reported that the exosomes with a hydrodynamic radius of $55 \sim 65 \mathrm{~nm}$ were rich in miRNAs, and the majority form of miRNAs was not mature but pre-miRNAs [29]. Pre-miRNAs are also able to exert biological effects after the conversion into mature miRNAs. The loading and secretion of miRNAs in exosomes have been ascertained as a strictly controlled process dependent on the source and developmental stage of derived cells rather than a random process.

Recent studies have clearly clarified that miRNAs mediate biological effects of exosomes on a variety of tissues [45, 49]. For example, MSCs-derived exosomes lead to a significant inhibition of angiogenesis through miR-16-mediated downregulation of VEGF in breast cancer cells [48]. Gastric cancer tissue-derived mesenchymal stem cells were shown to promote growth and migration of gastric cancer via transferring exosomal miR-221 [45]. In cardiovascular system, miRNAs-bearing exosomes or microvesicles are readily internalized into CMCs and ECs, resulting in cardiomyocyte protection and angiogenesis promotion [49-50]. These studies uncover important roles of miRNAs encapsulated in exosomes in the development of CVDs.

\section{Effects of exosomes on cardiovascular system}

\subsection{Cardiac benefits of exosomes}

\section{Anti-apoptosis}

Myocardial infarction is characterized by progressive loss of CMCs consequently resulting in congestive heart failure (CHF), and has become a difficult challenge due to the failure of CMCs to replace apoptotic cells. Emergence of MSCs provides a novel approach for therapeutics of MI not only because of their capability to directly differentiate into CMCs but also their anti-apoptotic effect from paracrine action [49, 51-52].

Recent study has reported that MSCs had ability to ameliorate ischemic CMCs injury by transferring miR-22 in exosomes targeting methyl CpG binding protein 2 (Mecp2) to reduce apoptosis [52] (Fig. 2).
Moreover, delivery of miR-221 in exosomes mediated anti-apoptotic effect of MSCs by inhibiting p53-upregulated modulator of apoptosis (PUMA), a subclass of the Bcl-2 protein family [49]. PUMA was shown to interplay with BCL-xL and p53 [53], and activate pro-apoptotic proteins while restrain anti-apoptotic proteins, so inhibition of PUMA by miR-221 could give rise to CMCs survival [49]. These findings suggest that miRNAs-bearing exosomes are involving in anti-apoptotic process.

\section{Anti-inflammatory effect}

It is well known that exosomes from dendritic cells, MHC class II+ cells and tumor cells aggressively participate in inhibition of inflammation response, which is considered as a novel therapeutic approach for some diseases [54-56]. Likewise, some studies have also proved that exosomes derived from MSCs indeed produce similar benefits on CVDs [57-58]. It was revealed that suppression of inflammation by MSCs-derived exosomes in lungs accords for relieving symptoms of pulmonary hypertension (PH) [57]. Additionally, it was also reported that MSCs-secreting exosomes contributed to a smaller number of white blood cell count and alleviated inflammation reaction accompanied by decreased infarct size and enhanced cardiac function in hearts after ischemia-reperfusion injury (IRI) [58] (Fig. 2). These studies reveal MSCs-derived exosomes exert protective effects against CVDs through inhibition of inflammation reaction.

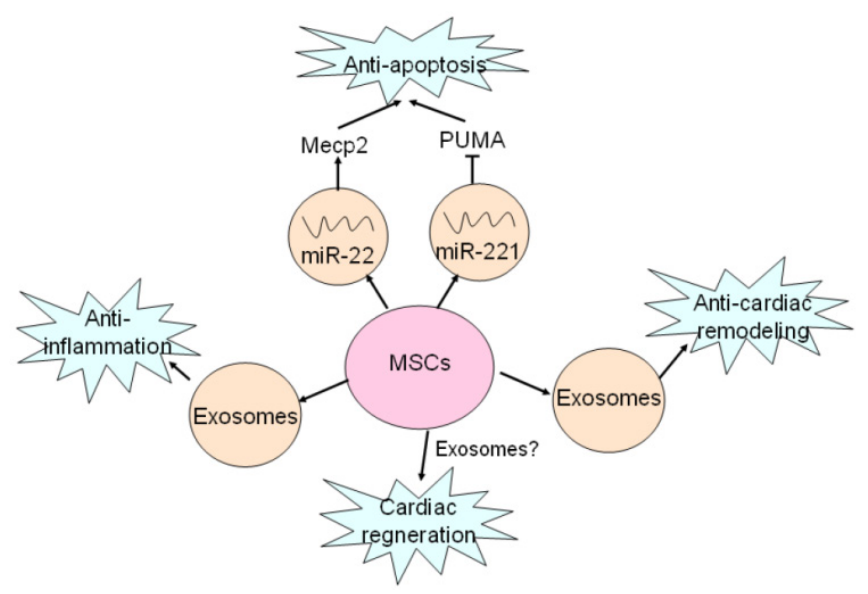

Fig. 2. Exosomes mediates beneficial effects of MSCs on hearts. MSCs secret exosomes containing miR-22 and miR-221 to target Mecp2 and PUMA, and thus exert anti-apoptotic effects. Anti-inflammatory effect of MSCs is mediated by exosomes as well as MVs. Exosomes are also responsible for anti-cardiac remodeling of MSCs. Cardiac regeneration property of MSCs has been proved, but whether exosomes are involved in this process remains unclear. 


\section{Anti-cardiac remodeling}

Cardiac remodeling, a compensatory consequence of CVDs such as myocardial hypertrophy and fibrosis, usually develops into heart failure without proper treatments [59]. Autologous MSCs transplantation has been shown to reduce infarct size, improve left ventricular ejection fraction (LVEF) and reverse remodeling after chronic myocardial infraction [60]. Recent study uncovered that exosomes derived from MSCs was able to enhance myocardial viability and prevent adverse remodeling after myocardial ischemia-reperfusion injury via activating pro-survival signaling, restoring bioenergetics and reducing oxidative stress [58]. Though anti-cardiac remodeling effect of MSCs-derived exosomes was confirmed, mechanisms of exosomes-mediated protection have not been verified.

\section{Cardiac regeneration}

Necrosis and apoptosis of CMCs due to pathological microenvironment or damaged factors is causal to heart failure while conventional therapies such as medications, surgeries and even intervention have certain limitations. Cardiac regeneration by MSCs and cardiac stem cells (CSCs) differentiation is raised as a novel strategy for curing CVDs [61-63]. It has been found that MSCs had ability to induce endogenous cardiac regeneration [28] (Fig. 2). MVs and exosmes released by cardiomyocyte progenitor cells (CPCs) could induce cardiac regeneration and improve cardiac function by promoting migration of ECs and secretion of VEGF resulting in angiogenesis [64-65]. The combination of human MSCs and human cardiac stem cells have a better impact on the reduction of infarct size and improve cardiac functions than MSCs alone [66]. However, whether exosomes take part in cardiac regeneration of MSCs still remains unclear, and the role of exosomes in cardiac regeneration needs further investigations.

\subsection{Vascular benefits of exosomes}

\section{Neovascularization}

Neovascularization is one of multiple effects of exosomes involving arteriogenesis, vasculogenesis and angiogenesis which are associated with proliferation and migration of ECs and VSMCs, differentiation into ECs and VSMCs, and formation of ECs from formerly existing vessels [28, 67]. Exosomes from placental mesenchymal stem cells (pMSCs) induced by hypoxia are major participator in facilitating migration and vascularization of placental microvascular endothelial cells (PMEC) [67]. Adipose mesenchymal stem cells (ASCs) can secret extracellular vesicles including both exosomes and microvesicles, which are critical for angiogenesis both in vitro and in vivo following internalization by human microvascular endothelial cells (HMECs), while platelet-derived growth factor (PDGF) strengthens this positive influence by means of irritating ASCs to release exosomes and microvesicles which are rich in pro-angiogenic molecules [44] (Fig. 3). Exosomes released by human umbilical cord MSCs under hypoxic condition deliver mRNAs and miRNAs to target cells, and promote proliferation of umbilical cord endothelial cells (Fig. $3)$, therefore leading to angiogenic action, enhanced blood flow restoration and capillary network formation [50]. Exosomes secreted by MSCs are implicated in the stimulation of tumor growth via upregulating VEGF level in tumor cells and consequent angiogenic effect, but it also has been reported the property of MSCs-derived exosomes to inhibit angiogenic process in breast cancer tissues [48, 68]. Heterogeneity of MSCs, dosage of MSCs administration, tumor types and impurity of MSCs might be responsible for inverse impacts of MSCs-derived exosomes on the development of tumor [69].

\section{Anti-vascular remodeling}

Vascular remodeling is an adaptive reaction in response to hemodynamic changes involving hyperplasia, hypertrophy, apoptosis and migration of vascular cells as well as generation and degradation of extracellular matrix [70-74]. Some documents reported that paracrine activity is responsible for anti-vascular remodeling effect of MSCs in rats suffering from pulmonary hypertension by inhibiting Smad2, which is indicated by reduced pulmonary arterial pressure and symptoms amelioration [72]. Notably, MSCs-derived exosomes have been recently shown to block expression of hypoxia-induced mitogenic factor (HIMF) induced by hypoxia to inhibit proliferation, and it suggests MSCs-derived exosomes play an essential role in anti-vascular remodeling [57,73-74] (Fig. 3). But molecular mechanisms of anti-vascular remodeling effect of MSCs-derived exosomes need to be defined precisely.

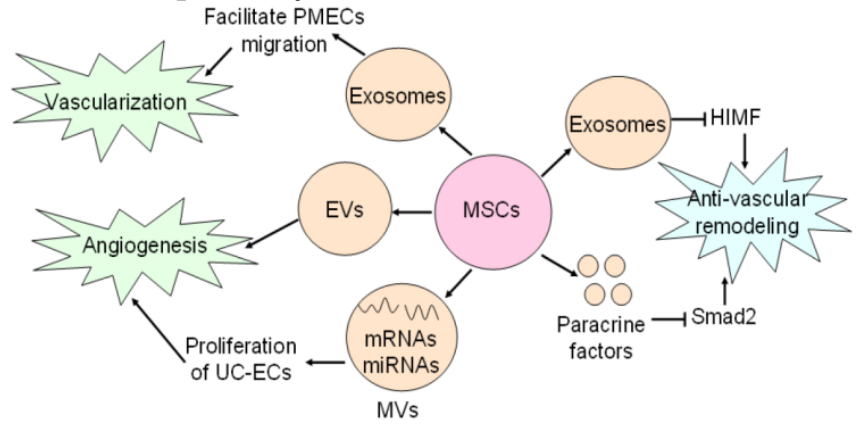

Fig. 3. Effects of MSCs on vasculature. MSCs secrete exosomes to facilitate PMECs migration, and thus contribute to vascularization. Angiogenic action of MSCs is mediated by MSCs-secreting EVs and MVs. MSCs generate exosomes and paracrine factors to inhibit HIMF and Smad2, and exert anti-vascular remodeling effect. 


\subsection{Roles of exosomes in MSCs therapy of CVDs}

\section{Myocardial infarction (MI)}

MI, a detrimental consequence of acute or persistent coronary occlusion, is featured by myocardial necrosis and cardiomyocyte loss [75]. It has been revealed that MSCs transplantation into infarcted region is able to improve cardiac function, and this efficacy could be enhanced in the case of MSCs modification with upregulation of miR-1 by promoting MSCs survival and differentiation into CMCs [76]. Interestingly, exosomes derived from MSCs contain the increased amount of miR-22 under ischemic condition, and can be centralized to CMCs to protect against apoptosis by miR-22 targeting Mecp2. Moreover, cardiac fibrosis is also antagonized by exosomes, which indicates exosomes-mediating critical protection for MI [52]. So, it was proposed that there exists a feed-forward loop network mediated by exosomes between ischemic hearts and other tissues: exosomes released by damaged myocardium transfer proteins and miRNAs which include ischemic signal to distant tissues or organs such as bone marrow (BM), leading to changes in the microenvironment of $\mathrm{BM}$ after MI; At the same time, exosomes from adjusted BM and progenitor cells are also delivered back to ischemic regions to trigger regeneration and repair processes [77].

\section{Reperfusion injury}

It is well documented that ischemia-reperfusion may result in irreversible structural damage and dysfunction of organs, which would be greatly attributed to generation of excessive free radicals [78]. In spite of Vitamin E and its analogues discovered as ideal antioxidants against ischemia-reperfusion injury, few studies refer to relevant applications in clinical trials [79-81]. Hopefully, great interest has arisen in the consideration of MSCs as a prospective agent for ischemia-reperfusion injury due to involvement of
MSCs in both myogenesis and angiogenesis to generate protective effects including reduction of infarct size, remarkable amelioration of left ventricular performance and increased amount of capillary vessels [82]. Intact exosomes secreted by MSCs were found to reduce oxidative stress, increase ATP and NADH, control inflammatory activities and activate PI3K/ Akt pathway, leading to protective influences on CMCs survival and retention of left ventricular function after ischemia-reperfusion injury, which indicates exosomes as a supplemental entity for ischemia-reperfusion therapy [58].

\section{Pulmonary hypertension}

$\mathrm{PH}$ is a kind of malignant pulmonary vascular diseases and characterized by an increase in pulmonary artery pressure, which may lead to right heart failure and even death. Pulmonary inflammation induced by hypoxia and bronchopulmonary dysplasia induced by hyperoxia may account for the development of PH [57, 83]. Administration of MSCs has been proved to confer a better prognosis of $\mathrm{PH}$ than medicines applied in clinics now, and has been widely tested in $\mathrm{PH}$ rats to uncover its underlying mechanisms [84-85].

$\mathrm{PH}$ following pulmonary vascular remodeling is highly correlated with pulmonary inflammation caused by hypoxia [57, 86]. MSCs-derived exosomes (MEX) treatment directly suppress activation of hypoxic signal pathway and then result in interference with alternative activated alveolar macrophages by targeting Th1/Th2 balance and down-regulation of proliferative miR-17 superfamily by shifting the balance of proliferation to inhibit PH. Moreover, MEX might disturb STAT3-miR-204-STAT3 feedback to ameliorate vascular remodeling. The findings above elucidate that MEX are protective for $\mathrm{PH}$ through the suppression of early pulmonary inflammation and vascular remodeling [57].

Table 1. Origins and functions of exosomes derived from mesenchymal stem cells.

\begin{tabular}{|c|c|c|c|}
\hline Exosomes & Cells & microRNAs/Target Genes & Functions \\
\hline Exosomes [52] & MSCs & miR-22/Mecp2 & $\begin{array}{l}\text { Reduce apoptosis and ameliorate ischemic } \\
\text { CMCs injury }\end{array}$ \\
\hline Exosomes [49] & MSCs & miR-221/PUMA & $\begin{array}{l}\text { Reduce CMCs apoptosissis and enhance CMCs } \\
\text { survival }\end{array}$ \\
\hline Exosomes [67] & pMSCs & unknown & Facilitate PMECs migration and vascularization \\
\hline Exosomes [44] & ASCs & unknown & Angiogenesis \\
\hline Exosomes [68] & MSCs & unknown & $\begin{array}{l}\text { Increase the level of VEGF and promote } \\
\text { angiogenesis }\end{array}$ \\
\hline Exosomes [58] & MSCs & unknown & $\begin{array}{l}\text { Inhibit oxidative stress, activate PI3K/Akt } \\
\text { pathway and reduce inflammatory activities }\end{array}$ \\
\hline Exosomes [57] & MSCs & unknown & $\begin{array}{l}\text { Suppress the activation of hypoxic signal } \\
\text { pathway }\end{array}$ \\
\hline
\end{tabular}




\section{Exosomes as a new therapeutic strategy for CVDs}

The above studies reveal that MSCs-derived exosomes play an essential role in MSCs-based therapy of CVDs including MI, reperfusion injury and $\mathrm{PH}$. The detailed mechanisms underlying benefits of exosomes in MSCs transplantation of these CVDs require further investigations. Administration of exosomes from MSCs instead of MSCs transplantation has displayed therapeutic potential on CVDs including increasing survival of cardiomyocytes as well as to inhibiting apoptosis. Thus, exosomes derived from MSCs represents a promising approach for the treatment of CVDs [58]. Compared to traditional MSCs therapies, MSCs-exosomes therapies will decrease injury from MSCs transplantation surgery, avoid the risk of unexpected differentiation into other cell types such as osteoblasts, adipocytes and chondrocytes as well as vascular calcification, and reduce possibility of favoring tumor growth by MSCs. Accordingly, administration of in vitro purified MSCs-derived exosomes becomes more attractive than conventional stem cells transplantation. Effects of MSCs-derived exosomes on cardiovascular system should be largely attributed to functional cytokines, miRNAs and proteins in exosomes. Nevertheless, how to preserve biological activity of cytokines, miRNAs and proteins in exosomes and deliver them to target sites is a big challenge for us now [30]. Following investigations are needed to solve the troubles of MEX therapy and make MEX become a promising entity for CVDs.

\section{Conclusions}

Much interest has been generated in exploring the role of exosomes in stem cell-based therapy of CVDs since they were discovered. Transferring characteristic of exosomes suggests its involvement in regulating cell-to-cell communication [87-89]. Notably, the possibility of exosomes as a diagnostic biomarker in clinics has been proposed due to their derived cell developmental stage-dependent and source-dependent features [90]. Though MEX possess more advantages than MSCs for CVDs therapy, further investigations are required to explore detailed mechanisms of generation, secretion, shuttle and action of MSCs-derived exosomes. Exosomes will be a new promising therapeutic strategy for CVDs in the future.

\section{Abbreviations}

CVDs: Cardiovascular diseases; MI: Myocardial infarction; MSCs: Mesenchymal stem cells; CMCs: Cardiomyocytes; ECs: Endothelial cells; VSMCs: Vascular smooth muscle cells; pre-miRNAs: Precur-
sor-microRNAs; pri-miRNAs: Primary miRNAs; miRNAs: MicroRNAs; RISC: RNA-induced silencing complex; VEGF: Vascular endothelial growth factor; SMCs: Smooth muscle cells; CHF: Congestive heart failure; Mecp2: Methyl CpG binding protein 2; PUMA: p53 upregulated modulator of apoptosis; MEX: MSCs-derived exosome; PH: Pulmonary hypertension; MVs: Microvesicles; IRI: Ischemia-reperfusion injury; LVEF: Left ventricular ejection fraction; CSCs: Cardiac stem cells; CPCs: Cardiomyocyte progenitor cells; pMSCs: Placental mesenchymal stem cells; PMECs: Placental microvascular endothelial cells; EVs: Extracellular vesicles; ASCs: Adipose mesenchymal stem cells; HMECs: Human microvascular endothelial cells; PDGF: Platelet-derived growth factor; HIMF: Hypoxia-induced mitogenic factor; BM: Bone marrow; IL-8: Interleukin-8.

\section{Acknowledgements}

This work was supported by the National Natural Science Fund of China (30900601/81170096), the Program for New Century Excellent Talents In Heilongjiang Provincial University (1252-NCET-013).

\section{Competing Interests}

The authors have declared that no competing interest exists.

\section{References}

1. Christoforou N, Gearhart JD. Stem cells and their potential in cell-based cardiac therapies. Prog Cardiovasc Dis. 2007; 49: 396-413. doi: 10.1016/j.pcad.2007.02.006

2. Wu JY, Liao C, Xu ZP, Chen JS, Gu SL. [A modified method to isolate and identify the adult mesenchymal stem cells from human bone marrow]. Zhongguo Shi Yan Xue Ye Xue Za Zhi. 2006; 14: 557-60.

3. Mazzetti MP, Oliveira IS, Miranda-Ferreira R, Fauaz G, Ribeiro CN, Gomes $\mathrm{PO}$, et al. Qualitative and quantitative analysis of rabbit's fat mesenchymal stem cells. Acta Cir Bras. 2010; 25: 24-7.

4. Zhang MW, Guo ZK, Liu XD, Wu Y, Hou CM, Mao N. [Development of methodology for isolation and culture of mesenchymal stem cells derived from mouse skeletal muscle]. Zhongguo Shi Yan Xue Ye Xue Za Zhi. 2003; 11: 538-41. doi:1009-2137(2003)05-0538-04.

5. Ling L, Ni Y, Wang Q, Wang H, Hao S, Hu Y, et al. Transdifferentiation of mesenchymal stem cells derived from human fetal lung to hepatocyte-like cells. Cell Biol Int. 2008; 32: 1091-8. doi:10.1016/j.cellbi.2008.04.020.

6. Gotherstrom C, Ringden O, Westgren M, Tammik C, Le Blanc K. Immunomodulatory effects of human foetal liver-derived mesenchymal stem cells. Bone Marrow Transplant. 2003; 32: 265-72. doi:10.1038/sj.bmt.1704111.

7. Cao H, Chu Y, Zhu H, Sun J, Pu Y, Gao Z, et al. Characterization of immortalized mesenchymal stem cells derived from foetal porcine pancreas. Cell Prolif. 2011; 44: 19-32. doi:10.1111/j.1365-2184.2010.00714.x.

8. Lee JC, Min HI, Park HJ, Lee S, Seong SC, Lee MC. Synovial membrane-derived mesenchymal stem cells supported by platelet-rich plasma can repair osteochondral defects in a rabbit model. Arthroscopy. 2013; 29: 1034-46. doi:10.1016/j.arthro.2013.02.026.

9. Rust PA, Kalsi P, Briggs TW, Cannon SR, Blunn GW. Will mesenchymal stem cells differentiate into osteoblasts on allograft? Clin Orthop Relat Res. 2007; 457: 220-6. doi:10.1097/BLO.0b013e31802e7e8f.

10. Morganstein DL, Wu P, Mane MR, Fisk NM, White R, Parker MG. Human fetal mesenchymal stem cells differentiate into brown and white adipocytes: a role for ERRalpha in human UCP1 expression. Cell Res. 2010; 20: 434-44. doi: 10.1038/cr.2010.11.

11. Zhang X, Hirai M, Cantero S, Ciubotariu R, Dobrila L, Hirsh A, et al. Isolation and characterization of mesenchymal stem cells from human umbilical cord blood: reevaluation of critical factors for successful isolation and high ability to proliferate and differentiate to chondrocytes as compared to mesenchymal 
stem cells from bone marrow and adipose tissue. J Cell Biochem. 2011; 112: 1206-18. doi:10.1002/jcb.23042.

12. Kawada H, Fujita J, Kinjo K, Matsuzaki Y, Tsuma M, Miyatake H, et al. Nonhematopoietic mesenchymal stem cells can be mobilized and differentiate into cardiomyocytes after myocardial infarction. Blood. 2004; 104: 3581-7. doi:10.1182/blood-2004-04-1488.

13. Xu Y, Meng H, Li C, Hao M, Wang Y, Yu Z, et al. Umbilical cord-derived mesenchymal stem cells isolated by a novel explantation technique can differentiate into functional endothelial cells and promote revascularization. Stem Cells Dev. 2010; 19: 1511-22. doi:10.1089/scd.2009.0321.

14. Silva GV, Litovsky S, Assad JA, Sousa AL, Martin BJ, Vela D, et al. Mesenchymal stem cells differentiate into an endothelial phenotype, enhance vascular density, and improve heart function in a canine chronic ischemia model. Circulation. 2005; 111: 150-6. doi:10.1161/01.CIR.0000151812.86142.45.

15. Ventura C, Cantoni S, Bianchi F, Lionetti V, Cavallini C, Scarlata I, et al. Hyaluronan mixed esters of butyric and retinoic Acid drive cardiac and endothelial fate in term placenta human mesenchymal stem cells and enhance cardiac repair in infarcted rat hearts. J Biol Chem. 2007; 282: 14243-52. doi: 10.1074/jbc.M609350200

16. Kurpinski K, Lam H, Chu J, Wang A, Kim A, Tsay E, et al. Transforming growth factor-beta and notch signaling mediate stem cell differentiation into smooth muscle cells. Stem Cells. 2010; 28: 734-42. doi:10.1002/stem.319.

17. Liu J, Li W, Wang Y, Fan W, Li P, Lin W, et al. Islet-1 overexpression in human mesenchymal stem cells promotes vascularization through monocyte chemoattractant protein-3. Stem Cells. 2014; 32: 1843-54. doi:10.1002/stem.1682.

18. Dufourcq P, Descamps B, Tojais NF, Leroux L, Oses P, Daret D, et al. Secreted frizzled-related protein-1 enhances mesenchymal stem cell function in angiogenesis and contributes to neovessel maturation. Stem Cells. 2008; 26: 2991-3001. doi:10.1634/stemcells.2008-0372.

19. Suzuki S, Narita Y, Yamawaki A, Murase Y, Satake M, Mutsuga M, et al. Effects of extracellular matrix on differentiation of human bone marrow-derived mesenchymal stem cells into smooth muscle cell lineage: utility for cardiovascular tissue engineering. Cells Tissues Organs. 2010; 191: 269-80. doi: $10.1159 / 000260061$.

20. Quevedo HC, Hatzistergos KE, Oskouei BN, Feigenbaum GS, Rodriguez JE, Valdes D, et al. Allogeneic mesenchymal stem cells restore cardiac function in chronic ischemic cardiomyopathy via trilineage differentiating capacity. Proc Natl Acad Sci U S A. 2009; 106: 14022-7. doi:10.1073/pnas.0903201106.

21. Mangi AA, Noiseux N, Kong D, He H, Rezvani M, Ingwall JS, et al. Mesenchymal stem cells modified with Akt prevent remodeling and restore performance of infarcted hearts. Nat Med. 2003; 9: 1195-201. doi:10.1038/nm912.

22. Tang $Y L$, Zhao $Q$, Qin $X$, Shen $L$, Cheng $L, G e ~ J$, et al. Paracrine action enhances the effects of autologous mesenchymal stem cell transplantation on vascular regeneration in rat model of myocardial infarction. Ann Thorac Surg. 2005: 80: 229-36: discussion 36-7. doi:10.1016/j athoracsur. 2005.02.072.

23. Van Overstraeten-Schlogel N, Beguin Y, Gothot A. Role of stromal-derived factor-1 in the hematopoietic-supporting activity of human mesenchymal stem cells. Eur J Haematol. 2006; 76: 488-93. doi:10.1111/j.1600-0609.2006.00633.x.

24. Togel F, Hu Z, Weiss K, Isaac J, Lange C, Westenfelder C. Administered mesenchymal stem cells protect against ischemic acute renal failure through differentiation-independent mechanisms. Am J Physiol Renal Physiol. 2005; 289: F31-42. doi:10.1152/ajprenal.00007.2005.

25. Haider H, Jiang S, Idris NM, Ashraf M. IGF-1-overexpressing mesenchymal stem cells accelerate bone marrow stem cell mobilization via paracrine activation of SDF-1alpha/CXCR4 signaling to promote myocardial repair. Circ Res. 2008; 103: 1300-8. doi:10.1161/CIRCRESAHA.108.186742.

26. Tang J, Wang J, Guo L, Kong X, Yang J, Zheng F, et al. Mesenchymal stem cells modified with stromal cell-derived factor 1 alpha improve cardiac remodeling via paracrine activation of hepatocyte growth factor in a rat model of myocardial infarction. Mol Cells. 2010; 29: 9-19. doi:10.1007/s10059-010-0001-7.

27. Lai RC, Arslan F, Lee MM, Sze NS, Choo A, Chen TS, et al. Exosome secreted by MSC reduces myocardial ischemia/reperfusion injury. Stem Cell Res. 2010; 4: 214-22. doi:10.1016/j.scr.2009.12.003.

28. Wen Z, Zheng S, Zhou C, Yuan W, Wang J, Wang T. Bone marrow mesenchymal stem cells for post-myocardial infarction cardiac repair: microRNAs as novel regulators. J Cell Mol Med. 2012; 16: 657-71. doi:10.1111/j.1582-4934.2011.01471.x.

29. Chen TS, Lai RC, Lee MM, Choo AB, Lee CN, Lim SK. Mesenchymal stem cell secretes microparticles enriched in pre-microRNAs. Nucleic Acids Res. 2010; 38: 215-24. doi:10.1093/nar/gkp857.

30. Lai RC, Chen TS, Lim SK. Mesenchymal stem cell exosome: a novel stem cell-based therapy for cardiovascular disease. Regen Med. 2011; 6: 481-92. doi:10.2217/rme.11.35

31. Jenjaroenpun P, Kremenska Y, Nair VM, Kremenskoy M, Joseph B, Kurochkin IV. Characterization of RNA in exosomes secreted by human breast cancer cell lines using next-generation sequencing. PeerJ. 2013; 1: e201. doi:10.7717/peerj.201.

32. Yu X, Huang C, Song B, Xiao Y, Fang M, Feng J, et al. CD4+CD25+ regulatory $T$ cells-derived exosomes prolonged kidney allograft survival in a rat model. Cell Immunol. 2013; 285: 62-8. doi:10.1016/j.cellimm.2013.06.010.

33. Buschow SI, van Balkom BW, Aalberts M, Heck AJ, Wauben M, Stoorvogel W. MHC class II-associated proteins in B-cell exosomes and potential functional implications for exosome biogenesis. Immunol Cell Biol. 2010; 88: 851-6. doi: $10.1038 /$ icb. 2010.64
34. Rabesandratana H, Toutant JP, Reggio H, Vidal M. Decay-accelerating factor (CD55) and membrane inhibitor of reactive lysis (CD59) are released within exosomes during In vitro maturation of reticulocytes. Blood. 1998; 91: 2573-80.

35. Al-Nedawi K, Szemraj J, Cierniewski CS. Mast cell-derived exosomes activate endothelial cells to secrete plasminogen activator inhibitor type 1. Arterioscler Thromb Vasc Biol. 2005; 25: 1744-9. doi: 10.1161/01.ATV.0000172007.86541.76.

36. Heijnen HF, Schiel AE, Fijnheer R, Geuze HJ, Sixma JJ. Activated platelets release two types of membrane vesicles: microvesicles by surface shedding and exosomes derived from exocytosis of multivesicular bodies and alpha-granules. Blood. 1999; 94: 3791-9.

37. Wolfers J, Lozier A, Raposo G, Regnault A, Thery C, Masurier C, et al. Tumor-derived exosomes are a source of shared tumor rejection antigens for CTL cross-priming. Nat Med. 2001; 7: 297-303. doi:10.1038/85438.

38. Yang Y, Xiu F, Cai Z, Wang J, Wang Q, Fu Y, et al. Increased induction of antitumor response by exosomes derived from interleukin-2 gene-modified tumor cells. J Cancer Res Clin Oncol. 2007; 133: 389-99. doi:10.1007/s00432-006-0184-7.

39. Taverna S, Amodeo V, Saieva L, Russo A, Giallombardo M, De Leo G, et al. Exosomal shuttling of miR-126 in endothelial cells modulates adhesive and migratory abilities of chronic myelogenous leukemia cells. Mol Cancer. 2014; 13: 169. doi:10.1186/1476-4598-13-169.

40. Corrado C, Raimondo S, Saieva L, Flugy AM, De Leo G, Alessandro R. Exosome-mediated crosstalk between chronic myelogenous leukemia cells and human bone marrow stromal cells triggers an interleukin 8-dependent survival of leukemia cells. Cancer Lett. 2014; 348: 71-6. doi:10.1016/j.canlet.2014.03.009.

41. Yuan A, Farber EL, Rapoport AL, Tejada D, Deniskin R, Akhmedov NB, et al. Transfer of microRNAs by embryonic stem cell microvesicles. PLoS One. 2009; 4: e4722. doi:10.1371/journal.pone.0004722.

42. Valadi H, Ekstrom K, Bossios A, Sjostrand M, Lee JJ, Lotvall JO. Exosome-mediated transfer of mRNAs and microRNAs is a novel mechanism of genetic exchange between cells. Nat Cell Biol. 2007; 9: 654-9. doi:10.1038/ncb1596.

43. Ono M, Kosaka N, Tominaga N, Yoshioka Y, Takeshita F, Takahashi RU, et al. Exosomes from bone marrow mesenchymal stem cells contain a microRNA that promotes dormancy in metastatic breast cancer cells. Sci Signal. 2014; 7: ra63. doi:10.1126/scisignal.2005231.

44. Lopatina T, Bruno S, Tetta C, Kalinina N, Porta M, Camussi G. Platelet-derived growth factor regulates the secretion of extracellular vesicles by adipose mesenchymal stem cells and enhances their angiogenic potential. Cell Commun Signal. 2014; 12: 26. doi:10.1186/1478-811X-12-26.

45. Wang M, Zhao C, Shi H, Zhang B, Zhang L, Zhang X, et al. Deregulated microRNAs in gastric cancer tissue-derived mesenchymal stem cells: novel biomarkers and a mechanism for gastric cancer. Br J Cancer. 2014; 110: 1199-210. doi:10.1038/bjc.2014.14.

46. Xin H, Li Y, Cui Y, Yang JJ, Zhang ZG, Chopp M. Systemic administration of exosomes released from mesenchymal stromal cells promote functional recovery and neurovascular plasticity after stroke in rats. J Cereb Blood Flow Metab. 2013; 33: 1711-5. doi:10.1038/jcbfm.2013.152.

47. Wang J, Greene SB, Bonilla-Claudio M, Tao Y, Zhang J, Bai Y, et al. Bmp signaling regulates myocardial differentiation from cardiac progenitors through a MicroRNA-mediated mechanism. Dev Cell. 2010; 19. 903-12. doi:10.1016/j.devcel.2010.10.022.

48. Lee JK, Park SR, Jung BK, Jeon YK, Lee YS, Kim MK, et al. Exosomes derived from mesenchymal stem cells suppress angiogenesis by down-regulating VEGF expression in breast cancer cells. PLoS One. 2013; 8: e84256. doi:10.1371/journal.pone.0084256.

49. Yu B, Gong M, Wang Y, Millard RW, Pasha Z, Yang Y, et al. Cardiomyocyte protection by GATA-4 gene engineered mesenchymal stem cells is partially mediated by translocation of miR-221 in microvesicles. PLoS One. 2013; 8: e73304. doi:10.1371/journal.pone.0073304.

50. Zhang HC, Liu XB, Huang S, Bi XY, Wang HX, Xie LX, et al. Microvesicles derived from human umbilical cord mesenchymal stem cells stimulated by hypoxia promote angiogenesis both in vitro and in vivo. Stem Cells Dev. 2012; 21: 3289-97. doi:10.1089/scd.2012.0095.

51. Khubutiya MS, Vagabov AV, Temnov AA, Sklifas AN. Paracrine mechanisms of proliferative, anti-apoptotic and anti-inflammatory effects of mesenchymal stromal cells in models of acute organ injury. Cytotherapy. 2014; 16: 579-85. doi:10.1016/j.jcyt.2013.07.017.

52. Feng $Y$, Huang W, Wani M, Yu X, Ashraf M. Ischemic preconditioning potentiates the protective effect of stem cells through secretion of exosomes by targeting Mecp2 via miR-22. PLoS One. 2014; 9: e88685. doi:10.1371/journal.pone.0088685.

53. Follis AV, Chipuk JE, Fisher JC, Yun MK, Grace CR, Nourse A, et al. PUMA binding induces partial unfolding within BCL-xL to disrupt p53 binding and promote apoptosis. Nat Chem Biol. 2013. 9. 163-8 doi:10.1038/nchembio.1166.

54. Kim SH, Lechman ER, Bianco N, Menon R, Keravala A, Nash J, et al. Exosomes derived from IL-10-treated dendritic cells can suppress inflammation and collagen-induced arthritis. J Immunol. 2005; 174: 6440-8.

55. Kim SH, Bianco NR, Shufesky WJ, Morelli AE, Robbins PD. MHC class II+ exosomes in plasma suppress inflammation in an antigen-specific and Fas ligand/Fas-dependent manner. J Immunol. 2007; 179: 2235-41.

56. Teng H, Hu M, Yuan LX, Liu Y, Guo X, Zhang WJ, et al. Suppression of inflammation by tumor-derived exosomes: a kind of natural liposome packaged 
with multifunctional proteins. J Liposome Res. 2012; 22: 346-52. doi:10.3109/08982104.2012.710911.

57. Lee C, Mitsialis SA, Aslam M, Vitali SH, Vergadi E, Konstantinou G, et al. Exosomes mediate the cytoprotective action of mesenchymal stromal cells on hypoxia-induced pulmonary hypertension. Circulation. 2012; 126: 2601-11. doi:10.1161/CIRCULATIONAHA.112.114173.

58. Arslan F, Lai RC, Smeets MB, Akeroyd L, Choo A, Aguor EN, et al. Mesenchymal stem cell-derived exosomes increase ATP levels, decrease oxidative stress and activate PI3K/Akt pathway to enhance myocardial viability and prevent adverse remodeling after myocardial ischemia/reperfusion injury. Stem Cell Res. 2013; 10: 301-12. doi:10.1016/j.scr.2013.01.002.

59. Haizlip KM, Janssen PM. In vitro studies of early cardiac remodeling: impact on contraction and calcium handling. Front Biosci (Schol Ed). 2011; 3: 1047-57.

60. Schuleri KH, Feigenbaum GS, Centola M, Weiss ES, Zimmet JM, Turney J, et al. Autologous mesenchymal stem cells produce reverse remodelling in chronic ischaemic cardiomyopathy. Eur Heart J. 2009; 30: 2722-32. doi:10.1093/eurheartj/ehp265.

61. Tateishi K, Ashihara E, Takehara N, Nomura T, Honsho S, Nakagami T, et al. Clonally amplified cardiac stem cells are regulated by Sca-1 signaling for efficient cardiovascular regeneration. J Cell Sci. 2007; 120: 1791-800. doi:10.1242/jcs.006122

62. Sassoli C, Pini A, Mazzanti B, Quercioli F, Nistri S, Saccardi R, et al. Mesenchymal stromal cells affect cardiomyocyte growth through juxtacrine Notch-1/Jagged-1 signaling and paracrine mechanisms: clues for cardiac regeneration. J Mol Cell Cardiol. 2011; 51: 399-408. doi:10.1016/j.yjmcc.2011.06.004

63. Torella D, Ellison GM, Mendez-Ferrer S, Ibanez B, Nadal-Ginard B. Resident human cardiac stem cells: role in cardiac cellular homeostasis and potential for myocardial regeneration. Nat Clin Pract Cardiovasc Med. 2006; 3 Suppl 1: S8-13. doi:10.1038/ncpcardio0409.

64. Sabin K, Kikyo N. Microvesicles as mediators of tissue regeneration. Transl Res. 2014; 163: 286-95. doi:10.1016/j.trsl.2013.10.005.

65. Vrijsen KR, Sluijter JP, Schuchardt MW, van Balkom BW, Noort WA, Chamuleau SA, et al. Cardiomyocyte progenitor cell-derived exosomes stimulate migration of endothelial cells. J Cell Mol Med. 2010; 14: 1064-70. doi:10.1111/j.1582-4934.2010.01081.x.

66. Williams AR, Hatzistergos KE, Addicott B, McCall F, Carvalho D, Suncion V, et al. Enhanced effect of combining human cardiac stem cells and bone marrow mesenchymal stem cells to reduce infarct size and to restore cardiac function after myocardial infarction. Circulation. 2013; 127: 213-23. doi:10.1161/CIRCULATIONAHA.112.131110.

67. Salomon C, Ryan J, Sobrevia L, Kobayashi M, Ashman K, Mitchell M, et al. Exosomal signaling during hypoxia mediates microvascular endothelial cell migration and vasculogenesis. PLoS One. 2013; 8: e68451. doi:10.1371/journal.pone.0068451.

68. Zhu W, Huang L, Li Y, Zhang X, Gu J, Yan Y, et al. Exosomes derived from human bone marrow mesenchymal stem cells promote tumor growth in vivo. Cancer Lett. 2012; 315: 28-37. doi:10.1016/j.canlet.2011.10.002.

69. Klopp AH, Gupta A, Spaeth E, Andreeff M, Marini F, 3rd. Concise review: Dissecting a discrepancy in the literature: do mesenchymal stem cells support or suppress tumor growth? Stem Cells. 2011; 29: 11-9.

70. Dzau VJ, Gibbons GH. Vascular remodeling: mechanisms and implications. J Cardiovasc Pharmacol. 1993; 21 Suppl 1: S1-5.

71. Herity NA, Ward MR, Lo S, Yeung AC. Review: Clinical aspects of vascular remodeling. J Cardiovasc Electrophysiol. 1999; 10: 1016-24.

72. Xie J, Hu D, Niu L, Qu S, Wang S, Liu S. Mesenchymal stem cells attenuate vascular remodeling in monocrotaline-induced pulmonary hypertension rats. J Huazhong Univ Sci Technolog Med Sci. 2012; 32: 810-7. doi:10.1007/s11596-012-1039-x.

73. Wort SJ, Woods M, Warner TD, Evans TW, Mitchell JA. Endogenously released endothelin-1 from human pulmonary artery smooth muscle promotes cellular proliferation: relevance to pathogenesis of pulmonary hypertension and vascular remodeling. Am J Respir Cell Mol Biol. 2001; 25: 104-10. doi:10.1165/ajrcmb.25.1.4331.

74. Mao SZ, Fan XF, Xue F, Chen $R$, Chen XY, Yuan GS, et al Intermedin modulates hypoxic pulmonary vascular remodeling by inhibiting pulmonary artery smooth muscle cell proliferation. Pulm Pharmacol Ther. 2014; 27: 1-9. doi:10.1016/j.pupt.2013.06.004.

75. Feld S, Kjellgren O, Smalling RW. Aggressive interventional treatment of acute myocardial infarction. Lessons from the animal laboratory applied to the catheterization suite. Cardiology. 1995; 86: 365-73.

76. Huang F, Li ML, Fang ZF, Hu XQ, Liu QM, Liu ZJ, et al. Overexpression of MicroRNA-1 improves the efficacy of mesenchymal stem cell transplantation after myocardial infarction. Cardiology. 2013; 125: 18-30. doi:10.1159/000347081.

77. Sahoo S, Losordo DW. Exosomes and cardiac repair after myocardial infarction. Circ Res. 2014; 114: 333-44. doi:10.1161/CIRCRESAHA.114.300639.

78. Cannon RO, 3rd. Mechanisms, management and future directions for reperfusion injury after acute myocardial infarction. Nat Clin Pract Cardiovasc Med. 2005; 2: 88-94. doi:10.1038/ncpcardio0096.

79. Janero DR. Therapeutic potential of vitamin E against myocardial ischemic-reperfusion injury. Free Radic Biol Med. 1991; 10: 315-24.

80. Campo GM, Squadrito F, Campo S, Altavilla D, Quartarone C, Ceccarelli S, et al. Beneficial effect of raxofelast, an hydrophilic vitamin $\mathrm{E}$ analogue, in the rat heart after ischemia and reperfusion injury. J Mol Cell Cardiol. 1998; 30: 1493-503. doi: 10.1006/jmcc.1998.0713

81. Mickle DA, Weisel RD. Future directions of vitamin E and its analogues in minimizing myocardial ischemia-reperfusion injury. Can J Cardiol. 1993; 9: 89-93.

82. Tang J, Xie Q, Pan G, Wang J, Wang M. Mesenchymal stem cells participate in angiogenesis and improve heart function in rat model of myocardial ischemia with reperfusion. Eur J Cardiothorac Surg. 2006; 30: 353-61. doi:10.1016/j.ejcts.2006.02.070.

83. Hansmann G, Fernandez-Gonzalez A, Aslam M, Vitali SH, Martin T, Mitsialis $\mathrm{SA}$, et al. Mesenchymal stem cell-mediated reversal of bronchopulmonary dysplasia and associated pulmonary hypertension. Pulm Circ. 2012; 2: 170-81. doi:10.4103/2045-8932.97603.

84. Baber SR, Deng W, Master RG, Bunnell BA, Taylor BK, Murthy SN, et al Intratracheal mesenchymal stem cell administration attenuates monocrotaline-induced pulmonary hypertension and endothelial dysfunction. Am J Physiol Heart Circ Physiol. 2007; 292: H1120-8. doi:10.1152/ajpheart.00173.2006.

85. Luan Y, Zhang X, Kong F, Cheng GH, Qi TG, Zhang ZH. Mesenchymal stem cell prevention of vascular remodeling in high flow-induced pulmonary hypertension through a paracrine mechanism. Int Immunopharmacol. 2012; 14: 432-7. doi:10.1016/j.intimp.2012.08.001.

86. Jeffery TK, Morrell NW. Molecular and cellular basis of pulmonary vascular remodeling in pulmonary hypertension. Prog Cardiovasc Dis. 2002; 45: 173-202. doi:10.1053/pcad.2002.130041.

87. Mathivanan S, Ji H, Simpson RJ. Exosomes: extracellular organelles important in intercellular communication. J Proteomics. 2010; 73: 1907-20. doi:10.1016/j.jprot.2010.06.006.

88. Simons M, Raposo G. Exosomes--vesicular carriers for intercellular communication. Curr Opin Cell Biol. 2009; 21: 575-81. doi: 10.1016/j.ceb.2009.03.007.

89. Ludwig AK, Giebel B. Exosomes: small vesicles participating in intercellular communication. Int J Biochem Cell Biol. 2012; 44: 11-5. doi: 10.1016/j.biocel.2011.10.005.

90. Harding CV, Heuser JE, Stahl PD. Exosomes: looking back three decades and into the future. J Cell Biol. 2013; 200: 367-71. doi:10.1083/jcb.201212113. 